Objectives: Aim of the present study was to assess the prevalence of right (RV) or left ventricle (LV) systolic and/or diastolic dysfunction by standard echocardiography and tissue Doppler imaging (TDI).

Methods: Thirty patients with UCTD-risk-SSc (28 female, aged $47 \pm 13$ years, range $21-70$ ) and 30 age- and sex-matched controls underwent cardiac assessment by standard echocardiography and TDI.

Results: UCTD-risk-SSc patients and controls did not show any difference at standard echocardiographic evaluation. In particular, an inverted E/A ratio was pointed out in 10/30 patients and $7 / 30$ controls $(p=n s)$. TDI showed a mild impairment of $L V$ and RV diastolic $\left(E_{m} 15 \pm 4\right.$ vs $19 \pm 5, p<0.0001: E / E_{m} 6.1 \pm 1.7$ vs $4.8 \pm 1.2, p=0.001 ; E_{t} 14 \pm 3$ vs $16 \pm 2, p=0.02 ; E_{t} / A_{t} 0.9 \pm 0.4$ vs $1.3 \pm 0.3, p<0.002$; increased pulmonary artery wedge pressure $9 \pm 2$ vs $8 \pm 1, p=0.001$ )and systolic function $\left(S_{m} 13 \pm 3\right.$ vs $15 \pm 2 \mathrm{~cm} / \mathrm{sec}, p<0.0003 ; S_{t} 14 \pm 2$ vs $\left.16 \pm 3 \mathrm{~cm} / \mathrm{sec}, p<0.0001\right)$ in UCTD- risk-SSc patients in comparison to controls.

Conclusions: Our study shows that UCTD-risk-SSc patients present a previously unrecognized, mild biventricular systolic and diastolic dysfunction as compared to controls. The pathophysiologic meaning (i.e.intramyocardial artery disease and/or patchy fibrosis) as well the predictive value of developing overt SScin the short time await to be elucidated.

Disclosure of Interest: None declared

DOI: 10.1136/annrheumdis-2017-eular.3080

\section{FRI0379 PREVALENCE OF FAM111 B GENE MUTATIONS IN SYSTEMIC SCLEROSIS}

A. Gcelu ${ }^{1}$, G. Deshpande ${ }^{2}$, A.A. Kalla ${ }^{1}$, M. Tikly ${ }^{3}$, B. Mayosi ${ }^{2}$, B. Hodkinson ${ }^{1}$. ${ }^{1}$ Medicine, Division of Rheumatology; ${ }^{2}$ Cardiovascular Genetics Laboratory, University of Cape town, Cape Town; ${ }^{3}$ Medicine, Division of Rheumatology, University of Witwatersrand, Johannesburg, South Africa

Background: Systemic sclerosis (SSc) is a prototypic systemic fibrotic disease with unclearly characterized genetic basis. Implicated genes have been associated with autoimmune dysregulation with relatively few variants associated with fibrosis [1]. We have discovered that mutations in FAM111B gene cause hereditary fibrosing poikiloderma with tendon contractures, myopathy, and pulmonary fibrosis (POIKTMP)[2], a multisystem fibrotic condition with clinical aspects of SSc [3]. This observation has established FAM111B as a candidate gene for SSc. Objectives:

The objective is to investigate whether FAM111B gene mutations are present in SSc patients and further explore relationships between FAM111B mutations and clinical expression of SSc.

Methods: Patients with a definite diagnosis of SSc attending the Rheumatology outpatient departments at Groote Schuur Hospital, Cape Town, and Chris Hani Baragwanath Hospital, Johannesburg, were enrolled into the study. Physical examination assessing the extent of disease was done in all patients and the modified Rodnan skin score (mRSS) was used to determine the extent of the skin involvement. Blood samples were collected for DNA extraction and mutation screening using the high-resolution melt technique. Samples with abnormal electropherograms were selected for Sanger sequencing to identify mutations. Public databases were used to verify the frequency of variants in FAM111B.

Results: 131 patients were genotyped, 13 men and 118 women, with a mean age of 26.6 years and mean age of symptom onset at 25.3 years. The majority of patients were black (59.5\%). $72 \%$ of patients had diffuse systemic sclerosis (DSSc) with a median mRSS of 11 . Genetic analysis revealed seven rare genetic variants (C832G $>$ A; C855G $>$ T; C917A $>$ G; C937G $>$ A; C988C $>$ T; C995A>C and $C 1006 \mathrm{G}>\mathrm{C}$ ) in eight patients (five patients from Johannesburg and three patients from Cape Town) [table 1]. These variants were missense mutations of unknown significance with a minor allele frequency $<0.01$. No FAM111B mutations that cause POIKMT were found in patients with SSc.

Conclusions: Rare genetic variants of unknown significance (GVUS) in FAM111B gene were found in patients with SSc. It is possible that the GVUS may modify the function of $F A M 111 B$, and influence the pathogenesis of SSC or are rare polymorphisms with no functional impact.

\section{References:}

[1] Romano, E., et al., The genetics of systemic sclerosis: an update. Clin Exp Rheumatol, 2011. 29(2 Suppl 65): p. S75-86.

[2] Mercier, S., et al., Mutations in FAM111B cause hereditary fibrosing poikiloderma with tendon contracture, myopathy, and pulmonary fibrosis. Am J Hum Genet, 2013. 93(6): p. 1100-7.

[3] Khumalo, N.P., et al., Poikiloderma, tendon contracture and pulmonary fibrosis: a new autosomal dominant syndrome? Br J Dermatol, 2006. 155(5): p. 1057-61.

Disclosure of Interest: None declared

DOI: 10.1136/annrheumdis-2017-eular.6719

\section{FRI0380 PATIENT PREFERENCES AND DIFFICULTIES CONCERNING THE HOME TREATMENT OPTIONS IN SYSTEMIC SCLEROSIS (SSC)}

B. Pagnotelli ${ }^{1}$, C. Bruni ${ }^{1}$, S. Bellando Randone ${ }^{1}$, S. Guiducci ${ }^{1}$, G. Lepri ${ }^{1}$, J. Blagojevic $^{1}$, L. Rasero ${ }^{2}$, M. Matucci Cerinic ${ }^{1}$, D.E. Furst ${ }^{1,3},{ }^{1}$ Division of Rheumatology; ${ }^{2}$ Division of Research \& Development Clinical Practice,
AOUC, Florence, Italy; ${ }^{3}$ Division of Rheumatology, UCLA, Los Angeles, United States

Background: SSc patients suffer from Raynaud's phenomenon, hand skin hardening and scarring, digital ulcers, esophagus/gastric dysmotility, dysphagia and mucosal dryness. These symptoms significantly reduce patient's life autonomy and impair the capacity to handle their therapy usually made by different modalities of administration: oral, topical, inhalation, intramuscular, subcutaneous, intravenous, and rectal.

Objectives: to investigate the methods of administration used by SSc patients, their preferred methods, their compliance to pharmacological therapy, and the difficulties that are encountered by the patients during pharmacotherapy.

Methods: 2 questionnaires were prepared on an ad hoc basis. The first given to patients, the second to be filled out by the patient's physician. The first questionnaire comprised 25 questions to investigate the problems encountered by patients when taking their medications by correlating these with two validated activities indices: SHAQ-DI (activities of daily living) and the COCHIN scale (hand activities). The second questionnaire collected demographic and important SSc-related clinical information (eg.age, sex, disease duration, symptoms). 80 Ssc patients completed the questionnaires, maintaining anonymity. A "difficulty index" was also filled out, where: $0=$ no difficulty to $4=$ impossible to use or too difficult to use.

Results:

Table 1. Patient use of pharmacological therapies

\begin{tabular}{lcccc}
\hline & $\%$ Using & $\begin{array}{c}\text { \% Users preferring } \\
\text { this method }\end{array}$ & Difficult index & Comment \\
\hline Oral & 100 & 91,25 & 0,83 & Most preferred \\
Eye drops & 52,5 & 2,5 & 1,07 & Least preferred \\
Topicals & 33,75 & 3,75 & 0,77 & Cannot open the top (safety lock) \\
Injections & 28,25 & 2,5 & 2,17 & Cannot apply to skin \\
Inhaler & 16,25 & 0 & 1,07 & Cannot spray \\
Vaginal/rectal & 6,25 & 0 & 1,03 & \\
\hline
\end{tabular}

Table 2. Most frequent problems encountered

\begin{tabular}{lcccc}
\multicolumn{5}{l}{ Table 2. Most frequent problems encountered } \\
\hline & $\%$ Using & Most frequent problems & $\begin{array}{c}\text { Users have these } \\
\text { problems }\end{array}$ & Difficult index ${ }^{\dagger}$ \\
\hline Oral & 100 & Blister: pushing out pills & 73,75 & 2,22 \\
Eye drops & 52,5 & Open thin stoppers & 78,75 & 1,51 \\
Topicals & 33,75 & Cannot open the top (safety lock) & 70,5 & 1,4 \\
Inhaler & 16,25 & Cannot spray/push the inhaler & 85 & 1,71 \\
\hline
\end{tabular}

$\dagger 4=$ most difficult.

In particular, the dimension of the pills is problematic, and has identified a paradoxical situation because large pills are difficult to swallow $(41,25 \%$ patients who use pills) but at the same time small pills are difficult to pinch with the fingers (62,25\% patients who use pills).

Conclusions: SSc patients experience significant problems in maintaining adherence to treatments due to difficulties in the use of blisters and bottles with children proof stoppers. Pills still remain the most preferred method of treatment. In conclusion, patients unanimously wished to avoid the use of blisters, defintively preferring bottles without the children proof stopper to make the treatment easier and provide a more independent life.

Disclosure of Interest: None declared

DOI: 10.1136/annrheumdis-2017-eular.4848

\section{FRI0381 INCREASED RISK OF OSTEOPOROTIC FRACTURES IN ADULT PATIENTS WITH POLYMYOSITIS AND DERMATOMYOSITIS: A NATIONWIDE POPULATION-BASED COHORT STUDY}

C.-C. Lai ${ }^{1}$, W.-S. Chen ${ }^{1}$, Y.-P. Tsao ${ }^{1}$, Y.-S. Chang ${ }^{2} .{ }^{1}$ Division of Allergy, Immunology, and Rheumatology, Taipei Veterans General Hospital, Taipei City; ${ }^{2}$ Division of Allergy, Immunology and Rheumatology, Department of Internal Medicine, Shuang Ho Hospital, Taipei Medical University, New Taipei City, Taiwan, Province of China

Background: Patients with polymyositis and dermatomyositis (PM/DM) are characterized by chronic muscle weakness due to autoimmune-mediated myositis and are usually treated with corticosteroids initially. PM/DM patients prone to develop osteoporosis and subsequent fractures but are rarely investigated.

Objectives: To explore the incidence rate (IR) and risk factors of osteoporotic fractures (OFs) among adult PM/DM patients.

Methods: We conducted a cohort study by utilizing the Taiwan National Health Insurance database. PM/DM patients and respective age- and gender-matched cohort without PM/DM were enrolled. The primary endpoint was the initial event of OFs. We used the Cox proportional hazard model to study the risk factors of OFs in the PM/DM cohort.

Results: Among 2391 PM/DM patients (67.8\% female, mean age: 49.5 years) followed for a mean (SD) of 6.1 (5.0) years, 116 developed vertebral fractures, 32 had hip fractures, and 14 experienced radius fractures (IR: 8.18, 2.20, and 0.96 per 1000 person-years, respectively, Table 1). Compared with the matched cohort, the PM/DM patients had higher IR ratios (IRRs) (95\% Cls) of OFs at all age groups at enrollment: $3.27(2.19$ to $4.81, p<0.0001)$ for people $<50$ years and 2.29 ( 1.85 to $2.82, p<0.0001$ ) for those $\geq 50$ years. The IRRs were 2.39 (1.92 
to $2.94, p<0.0001)$ for vertebral fractures and $1.62(1.07$ to $2.38, p=0.0093)$ for hip fractures. PM/DM patients experienced vertebral fractures and hip fractures at younger ages (62.2 vs 68.4 and 66.0 vs 75.4 years, respectively; both $p<0.001$ ). Multivariable Cox regression analyses showed that being female gender, age $\geq 50$ years, having hypertension, coronary artery disease, asthma, and using daily prednisolone equivalent to $>5 \mathrm{mg}$ are associated with OFs.

Table 1. IRs and IRRs of osteoporotic fractures: overall and subgroup analysis

\begin{tabular}{lcccc}
\hline Subgroup & IM IR & Control IR & IRR $(95 \%$ CI $)$ & p Value \\
\hline Overall fracture & 10.90 & 5.23 & $2.08(1.73$ to 2.49$)$ & $<0.0001$ \\
Age $<50$ year & 4.51 & 1.38 & $3.27(2.19$ to 4.81$)$ & $<0.0001$ \\
Age $\geq 50$ year & 22.59 & 9.85 & $2.29(1.85$ to 2.82$)$ & $<0.0001$ \\
Vertebral fracture & 8.18 & 3.43 & $2.39(1.92$ to 2.94$)$ & $<0.0001$ \\
Age $<50$ year & 3.39 & 0.98 & $3.47(2.17$ to 5.44$)$ & $<0.0001$ \\
Age $\geq 50$ year & 16.82 & 6.33 & $2.66(2.07$ to 3.39$)$ & $<0.0001$ \\
Hip fracture & 2.20 & 1.36 & $1.62(1.07$ to 2.38$)$ & 0.0093 \\
Age $<50$ year & 0.65 & 0.10 & $6.56(1.75$ to 24.6$)$ & 0.0027 \\
Age $\geq 50$ year & 4.87 & 2.83 & $1.72(1.09$ to 2.62$)$ & 0.0081 \\
Radius fracture & 0.96 & 0.83 & $1.15(0.60$ to 2.02$)$ & 0.3081 \\
Age $<50$ year & 0.43 & 0.35 & $1.25(0.31$ to 3.69$)$ & 0.3289 \\
Age $\geq 50$ year & 1.86 & 1.40 & $1.35(0.62$ to 2.63$)$ & 0.1857 \\
\hline
\end{tabular}

$\mathrm{IR}$, incidence rate per 1000 person-year; IRR, incidence rate ratio; $\mathrm{Cl}$, confidence interval.

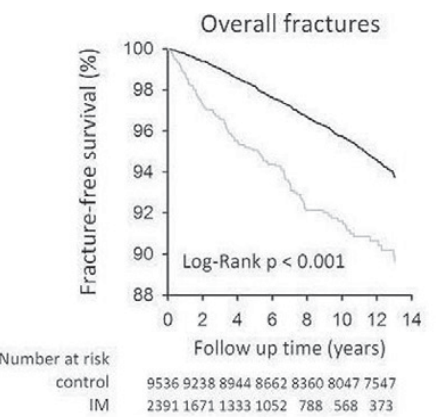

Vertebral fractures
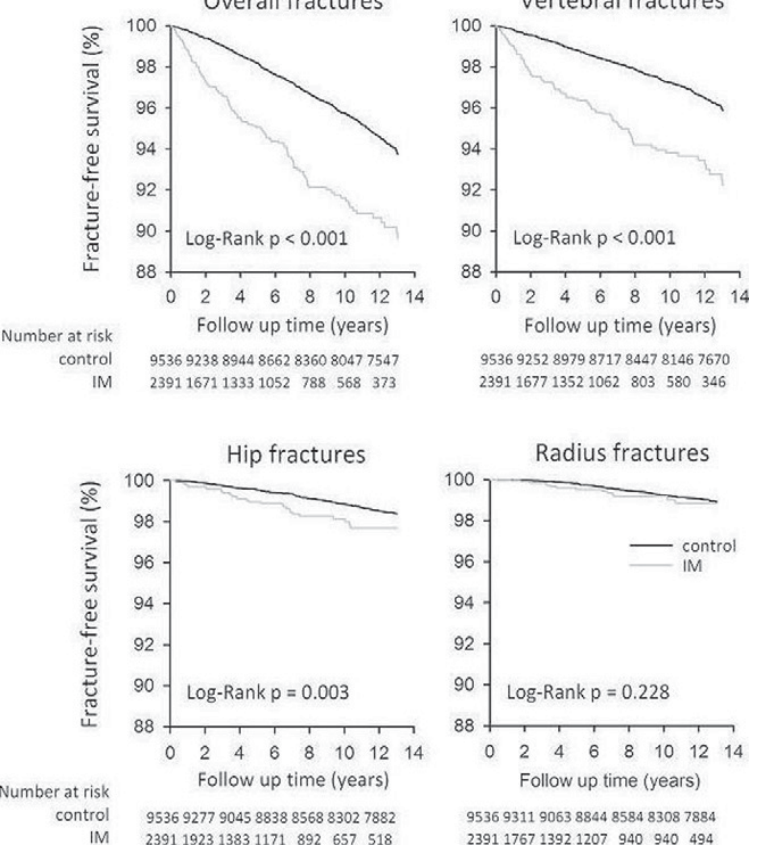

Conclusions: Adult PM/DM patients had a high IR of vertebral and hip fractures. Patients who were female, advanced age, having certain comorbidities, and exposed to corticosteroid exhibited a higher risk.

Disclosure of Interest: None declared

DOI: 10.1136/annrheumdis-2017-eular.6043

\section{FRI0382 USE OF THE DETECT ALGORITHM FOR EARLY PULMONARY ARTERY HYPERTENSION DIAGNOSIS IN PATIENTS WITH SYSTEMIC SCLEROSIS IN EVERYDAY CLINICAL PRACTICE}

C. Koutsianas, S. Subasinghe, K. Douglas. Department of Rheumatology, The Dudley Group NHS Foundation Trust, Dudley, United Kingdom

Background: The DETECT algorithm (DA) ${ }^{1}$ provides a sensitive tool for early identification of patients with Systemic Sclerosis (SSc) at risk of Pulmonary Arterial Hypertension (PAH) and advocates evaluation with Right Heart Catheterisation $(\mathrm{RHC})$. Few published data look into its implementation in everyday clinical practice and compare it to annual echocardiographic (TTE) screening.

Objectives: To compare DA and TTE screening of SSc patients for $\mathrm{RHC}$ referral rates and related patient characteristics.

Methods: Data on 57 consecutive patients with a diagnosis of SSc that had at least one visit to Russells Hall Hospital Scleroderma clinic from February to November 2016 was prospectively collected on Excel (clinical history, physical examination, immunological status, treatment, PFTs, NT-proBNP, urate, ECG, TTE, CXR and HRCT results). DETECT scores were calculated (www.detectpah.com) and compared with the TTE probability for PAH as per the 2015 ESC/ERS Guidelines².

Results: 31 patients with a full set of data were included in the final analysis. $93.5 \%$ were female with a mean age of $64.4 \pm 12.9$ years, $87 \%$ had limited cutaneous (Ic) SSc, $58.1 \%$ positive anticentromere antibody and mean time since diagnosis was $6.2 \pm 3.2$ years. The majority of patients $(80 \%)$ were asymptomatic for $\mathrm{PAH}$. Mean DLCO was $60.9 \pm 18.3 \%$ predicted (the DETECT study included on patients with $\mathrm{DLCO}<60 \%$ ). We did not exclude patients with renal insufficiency; mean eGFR was $74 \pm 17.7 \mathrm{ml} / \mathrm{min}$.

The implementation of DA recommended $\mathrm{RHC}$ in 18/31 patients (DETECT step 2 score $\geq 35,58 \%$, see table 1) compared to just $4 / 31$ (13\%) based on TTE by 2015 ESC/ERS guidelines. The additional 14 patients identified by DA were found to have no statistically significant differences in SSc type, dyspnea symptomatology, immunosuppressive treatment, presence of interstitial lung disease (ILD), age, time from diagnosis or symptom onset, eGFR and CRP compared to patients where RHC was not recommended. From the 18 patients identified by DA, only 4 reported shortness of breath. $2 / 18$ had existing cardiac conditions (aortic stenosis, left ventricular hypertrophy), while 9/18 had ILD (5 mild, 2 moderate, 2 severe as per HRCT).

\begin{tabular}{lcccc}
\hline \multicolumn{4}{c}{ Table 1 } & \multicolumn{3}{c}{ DETECT step 2 score } & \multirow{2}{*}{$p$} \\
\cline { 2 - 4 } & $<35$ & $35-39$ & $>40$ & \\
\hline Age, years (median (IQR)) & $65.8(26.9)$ & $63.2(22.3)$ & $72.4(25.1)$ & 0.875 \\
Disease duration, years (median (IQR)) & $8(4)$ & $4(7)$ & $5(9)$ & 0.996 \\
eGFR, ml/min (median (IQR)) & $80(28)$ & $83(17)$ & $54.5(47)$ & 0.958 \\
CRP, mg/L (median (IQR)) & $3(5)$ & $3(3)$ & $3(2)$ & 0.636 \\
DLCO, \% (median (IQR)) & $65(17)$ & $64(12)$ & $53(30)$ & 0.757 \\
ILD (no. of patients) No & 10 & 5 & 3 & 0.087 \\
$\quad$ Mild & 1 & 3 & 4 & \\
$\quad$ Moderate & 2 & 1 & 0 & \\
$\quad$ Severe & 0 & 0 & 2 & \\
\hline
\end{tabular}

Conclusions: Implementation of DA in a largely asymptomatic non-selected SSc patient population is more sensitive for $\mathrm{RHC}$ referral than annual TTE screening. Recommendations for RHC per DETECT score are difficult to predict on symptom and other traditional clinical parameters. The use of DA will lead to increased RHC referrals. Cardiologists may need education on this new method for screening and although the ESC/ERS 2015 guidelines discuss DA, they conclude that its cost-effectiveness has not been clarified as compared with symptom-based detection.

References:

[1] Coghlan JG et al. Evidence-based detection of pulmonary arterial hypertension in systemic sclerosis: the DETECT study. Ann Rheum Dis 2014 Jul;73 (7):1340-9.

[2] Galiè N et al. 2015 ESC/ERS Guidelines for the diagnosis and treatment of pulmonary hypertension. Eur Heart J (2015) 37 (1): 67-119.

Disclosure of Interest: None declared

DOI: 10.1136/annrheumdis-2017-eular.5928

\section{FRI0383 DETECT SCREENING FOR PULMONARY ARTERIAL HYPERTENSION IN SYSTEMIC SCLEROSIS: DATA FROM AN EUSTAR COHORT}

C. Ancuta ${ }^{1,2}$, C. Pomirleanu ${ }^{1,2}$, R. Maxim ${ }^{2}$, F. Mitu ${ }^{1,3} \cdot{ }^{1}$ University of Medicine and Pharmacy Grigore T Popa lasi; ${ }^{2}$ Rheumatology $2 ;{ }^{3}$ Cardiology, Clinical Rehabilitation Hospital, IASI, Romania

Background: Early documentation of pulmonary arterial hypertension (PAH) related to systemic sclerosis (SSc) remains a challenge in daily clinical practice, with major prognostic and therapeutic implications. Although several screening algorithms specifically designed to optimize the detection of secondary $\mathrm{PAH}$ have already been proposed (e.g., DETECT, REVEAL), no precise approach received complete validation, especially the use of invasive diagnostic right heart catheterization $(\mathrm{RHC})$.

Objectives: Main objective was to assess the risk of PAH in patients with SSc and to compare with PAH screening promoted by European Society of Cardiology/ European Respiratory Society (ESC/ERS) 2009 guidelines, while secondary one to identify predicting factors for developing $\mathrm{PAH}$ among clinical and immunological SSc variables.

Methods: Cross-sectional prospective single-center study, applying the DETECT calculator in patients with SSc recruited in EUSTAR 162 center cohort between January 2013- December 2016; all SSc completing at least two monitoring visits at 6 months were considered.

Standard assessments included MEDS (minimal data set EUSTAR), annual echocardiography with systolic pulmonary artery pressure (SPAP), pulmonary function tests with forced vital capacity (FVC), percentage predicted/diffusing capacity for carbon monoxide (DLCO) \% predicted, ECG, serum biomarkers (serum urate, NT-proBNP).

$\mathrm{PAH}$ risk calculator (http://detect-pah.com/pah-risk-calculator), a tool developed and validated in the DETECT study, is able to identify patients requiring echocardiography (STEP 1 ) respectively $\mathrm{RHC}$ (STEP 2 ), and was systematically applied in our cohort. All SPAP $\geq 45 \mathrm{mmHg}$ or between $35-45 \mathrm{mmHg}$ in the presence of dyspnea were proposed for invasive testing.

Statistical analysis was performed in IBM SPSS-19 version, $p<0.05$

Results: 41 out 56 SSc in our database were recruited: mainly women (85.36\%) with diffuse cutaneous SSc (63.41\%), anti-topoisomerase (up to half) and anticentromere (about one third) positive disease; one out of four 4 SSc presented with digital ulcers and one of three with active capillaroscopic pattern. 